\title{
INTERVIEW
}

For reprint orders, please contact: reprints@futuremedicine.com

\section{Aspects of modern breast cancer management: a surgeon's tale}

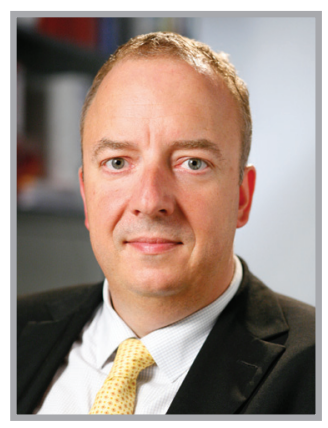

Michael Gnant*: Michael Gnant speaks to Marco De Ambrogi, Commissioning Editor: Michael Gnant is Full Professor of Surgery at the Medical University of Vienna, Austria, where he also serves as President of the Austrian Breast and Colorectal Cancer Study Group. His medical career began in 1988 when he graduated in medicine in Vienna. He then specialized in surgery and surgical oncology, working as a Visiting Scientist at the National Cancer Institute, NIH (MD, USA), then as a Full Professor at the Medical University of Vienna, where he also heads the Breast Health Center. His achievements include co-leading the Comprehensive Cancer Center Vienna and serving on the famous St. Gallen Consensus Panel for Early Breast Cancer since 2007, as well as being elected to the European Academy of Science and Arts in 2009. Among other honors, he is a Fellow of the American College of Surgeons. Gnant's research interests include several fields of surgical oncology, in particular breast and pancreatic cancer, immunotherapy using antibodies, vaccination with dendritic cells, endocrine intervention, dormant tumor cells, the use of bone-targeted treatments in order to silence tumor micrometastases, and pathway-directed therapies such as mTOR and CDK inhibition.

Q How did your medical career begin $\&$ where did your interest in breast cancer originate from?

I initially started my medical career as a transplant surgeon, and that interest in immunology and respective research eventually brought me to the field of cancer immunotherapy; in fact, in transplant immunology we aim to reach therapeutically what a cancer has already achieved: acquired, peripheral secondary tolerance. Moreover, the clinical aspects of being a cancer surgeon attracted me to that field: you are actually helping patients in one of the most stressful situations of their life, usually during or immediately after the diagnosis of a dangerous malignant disease, and this is both challenging and rewarding at the same time. Likewise, I try not to be just a 'cut-and-go' surgeon, meaning that I usually follow-up my cancer patients for prolonged, sometimes lifelong, periods of time. This is a much more holistic approach to human existence and caring for others than classical surgery, and my field of clinical expertise and research enables me to do exactly that.

I am involved in a number of surgical oncology fields, with particular focus on pancreatic and breast cancer (an unusual combination outside Japan, but at very different positions of the cancer surgery range). My interest in breast cancer originates from that fact that this disease is a pioneer field in the above-mentioned respects, and can serve as role model disease for all surgical oncology.

What research projects are you currently involved in?

Leading a large research group at my institution and coordinating a national clinical

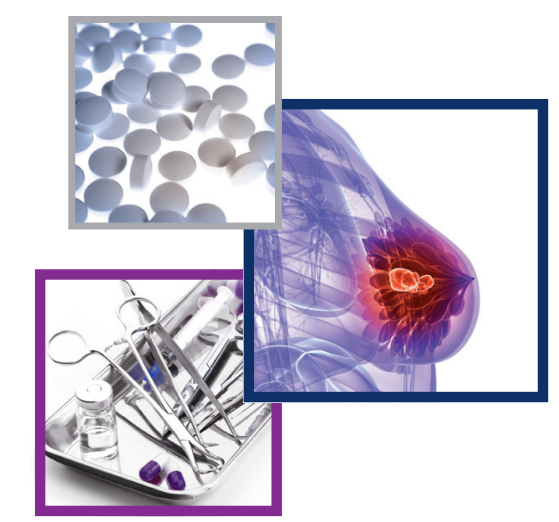

News \& Views

News

Journal Watch

Interview

*Department of Surgery, Medical University of Vienna, A-1090 Wien, Waehringer Guertel 18-20, Austria;

Tel.: +43 140400 5646; Fax: +43 140400 6807; michael.gnant@meduniwien.ac.at

Future 
trials group, as well as being involved in a variety of international research projects, it is not easy to nail this down to a particular sub-field. In postmenopausal breast cancer, we have just closed accrual to a large pivotal adjuvant trial (>3400 patients) of the anti-RANK-ligand denosumab, aiming at prevention of treatment-induced fractures, as well as at outcome improvements.

We are also currently doing a number of Phase-II-type neoadjuvant studies exploring the clinical potential of targeting the PI3K pathway. In the postneoadjuvant setting, we will start an international CDKinhibitor trial led by our friends at the German Breast Group; at the same time we aim at setting up a global network exploring this exciting option for a larger group of adjuvant breast cancer patients.

We have also just started AURORA, an international program of molecularly defined treatments of metastatic breast cancer, which is run by my international activity umbrella, the successful Breast International Group. In addition, we run a number of surgical and neoadjuvant trials in other cancers, including pancreatic cancer and colorectal liver metastases.

Q You have carried out much work on the use of bisphosphonates in breast cancer, how has this research progressed?

It is now almost 20 years since the antitumoral properties of experimental bisphosphonate therapy caught my attention. After a lot of reading and interactions with experts from the bone field, we decided that we could potentially explore this approach for two purposes: first, for maintaining bone health in patients receiving bone-compromising adjuvant therapies; and secondly, for exploiting some direct anti-tumor actions against tumor cells in early breast cancer. Apparently, the former goal was reached bisphosphonates have clearly been shown to protect bone from estrogen-lowering treatments by many groups, including us.

The latter issue is more complicated: there might be not too much of a direct anti-tumor effect of bisphosphonates, but we found indirect effects on the bone marrow microenvironment, which actually led to exciting outcome improvements. At least in postmenopausal women and young patients on therapeutic ovarian function suppression therapy, adjuvant zoledronic acid significantly improves disease-free and overall survival. Since we observe benefits, not only with respect to the prevention of bone metastases, but also in reduction in relapses outside the bone (including locoregional relapses), this hints at the validity of the century-old 'seed-and-soil' hypothesis: when we render the 'soil' - the bone marrow microenvironment in which dormant tumor (stem) cells survive - more hostile and less fertile, we can observe sustained clinical outcome benefits.

These observations, which started after the first presentation of the ABCSG-12 trials, which I was allowed to give in an ASCO highlight plenary session in 2008, are exciting in multiple ways: first, we have detected a rather tolerable and nonproblematic therapy that improved long-term breast cancer outcomes by $20-30 \%$. Second, and this is really reassuring to me, we can demonstrate that a relatively shortterm intervention (we gave treatment only for the first 3 years and then stopped) will show a sustained and persisting difference even at 8 or 10 years of follow-up. Furthermore, our knowledge and research about early cancer has been greatly stimulated by this study - in my opinion, eventually, the secrets for curing cancer will be found exactly in this area of research.

Q Your recent work has also focused on the use of aromatase inhibitors in pre- \& post-menopausal women: what can you tell us about this?

We have contributed with more than 10,000 patients to endocrine therapy trials in the last two decades. First ABCSG-8 and other trials have demonstrated that aromatase inhibitors are superior to the former standard tamoxifen. However, this advantage comes at the expense of some tolerability issues that can be annoying for patients in clinical practice. We are still unsure about what is the exact patient subgroup that will benefit the most, so, like it happens sometimes in oncology, we probably overtreat too many patients in order to be on the safe side of the outcome 
benefit. The situation is more complex in premenopausal patients: here, we have so far no perfect proof that aromatase inhibitors are superior to tamoxifen in the presence of ovarian function suppression therapy.

A very important question in endocrine therapy of postmenopausal breast cancer is treatment duration: we have been able to demonstrate with ABCSG-6a that just extending therapy with aromatase inhibitors for three additional years beyond the usual 5 years of endocrine therapy has significantly improved results. These results, taken together with similar results, for example from MA17 or ATLAS, leave us with the question of 'How long do we have to treat?' in the clinic. We have recruited 3500 patients for our ABCSG16/SALSA trial and randomized 2 versus 5 years of extended anastrozole - we will have to wait for a couple of more years for the results.

\section{Q What is your opinion on the} systematic use of chemotherapy \& gene array diagnostic tools in the clinic?

In general, we overtreat too many patients, particularly in hormone-receptor-positive disease. As a community, we need to accept that $1-2 \%$ improvement in the 10 -year survival rate does not translate into a worthy benefit for the average patient, unless we can identify better who will actually derive benefit and for whom we can spare the side effects of cytotoxic therapy (or any other treatment).

The great achievement of the currently available gene array diagnostics is that they have brought a lot of focus and attention to this most important field of oncology in general: individualizing treatment strategies. However, despite having been involved in the development of several RNA-based tools, we have to realize that the true value of these new tools (which are not exactly cheap) remains to be identified accurately, using proper clinical trial methodology. Personally, I am not too happy about the fact that these assays are sometimes used (and marketed) as simple recipes for treatment decisions; they are useful tools in some patients, but probably completely unnecessary in others.
Q Can you tell us why, in your experience, chemoprevention should be preferred to preventive mastectomy? With respect to chemoprevention, we have to clearly state that we are not there. Despite some encouraging results, and maybe the existence of a clinically relevant patient target group (certain high risk women) with respect to preventive endocrine approaches, we still cannot offer a pill that reduces breast cancer incidence for most women. Needless to say, this would be great if we could achieve a favorable benefit-side effect relationship.

The issue of surgical prevention is a different one, particularly if it involves mutilating procedures such as mastectomies. I am saddened by the fact that we now see increasing mastectomy rates in some places around the world despite having successfully fought to eliminate mastectomy for most breast cancer patients. I am not talking about preventive surgery in the setting of defined $B R C A$ families; I certainly think that in the rare situations of truly definable inherited risk, this option should be carefully considered. I cannot see an ethical or medical justification for preventive mastectomies outside of $B R C A$ families: some of these women may have been incompletely informed or sometimes even victimized by surgical marketing strategies. Considering preventive mastectomy without defined genetic risk is always a matter of fear and anxiety, and the treatment for these should never be surgery, but counseling and psychological support.

Even in our successful neoadjuvant therapies that enable breast conservation on many cases where the initial tumor size may have indicated mastectomy, there are too many unnecessary mastectomies. We have to educate better our professional colleagues, as well as our patients, about the fact that nowadays mastectomies are probably the inevitable surgical approach for not more than $10 \%$ of all breast cancer patients.

Q You have previously stressed the importance of shifting the role of the surgeon in a multidisciplinary approach to breast cancer: can you tell us more about that?

I am truly convinced that cancer surgery and cancer surgeons need to keep 
transforming from a monodisciplinary, sometimes lonesome and sometimes patronizing, discipline to the core of a multidisciplinary team: again, the field of breast cancer is pivotal for the whole of surgical oncology. This means change: in habits, attitude, but also in communication and logistical skills. If we surgeons fail to live up to the task, we risk reverting to a manual profession both in the clinic and in research.

Technical perfection in itself is not a particularly exciting goal for myself; it is quite obvious that we need to offer surgical procedures at the highest quality level and with minimized morbidity. I am thrilled by the new options and chances arising for patients by perfectly integrating surgery into the multimodality treatment process: being a surgeon by training may still help us to be effective, aim at a goal and guide the patient for whom we care through the process. Moreover, the role of the surgeon is increasing in importance in research since we need to integrate those who have access to repeated biomaterial collection, otherwise the multidisciplinary team is without alternative.

Q In your experience, how important for the final outcome are case manager approaches \& communication with breast cancer patients from diagnosis to post-treatment?

One potential drawback of the multidisciplinary approach that nowadays has to be the standard of care is that many people are involved. Several opinions and logistical challenges may have to be sorted out, and this poses the danger of losing time and eventually confusing the patient, who is waiting anxiously for treatment decisions. In order to avoid this, the role of a case manager is crucial. Most of all he/she needs to coordinate the process, but also communicate thoroughly and sensibly with the patient. In early breast cancer, the surgeon is best suited to fulfill this role in most healthcare environments and many excellent breast surgeons serve in this role perfectly.

Q You are President of the Austrian Breast \& Colorectal Study Group, which, among other aspects, is remarkable for the recruitment of a high number of participants to clinical trials: can you tell us which are the key factors in the success of the organization?

We have now recruited almost 25,000 patients to randomized clinical trials, which is remarkable in a small country of just 8 million inhabitants. What we have achieved through continuing collaboration over more than 25 years is that patients in Austria have understood the importance of contributing to clinical research. They don't feel like guinea pigs when asked to consent for participation; in fact, I have patients every week who just come to see me and ask: 'Do you have a clinical trial for my situation?'. This is a matter of transparency, good governance culture and ongoing communication efforts.

Moreover, the collaboration of physicians and other health professionals has been instrumental for ABCSG's success. In particular, for a small country such as Austria, it is important to be 'on the map' of international innovation and research development, because just as a market we may not be important. Thanks to more than 120 high-level publications, ABCSG has achieved this goal and will continue to strive for global excellence.

\section{Q You have previously expressed your} hopes for more state-funded research in breast cancer \& the creation of a European cancer research institute: do you see these two as something achievable in the near future?

In Europe, and particularly in Austria, publicly funded clinical research is severely lagging behind, as compared with the USA. Europe needs an equivalent to America's $\mathrm{NIH}$; it is probably unrealistic to have this in one location, but in a digital world, several institutions can be connected as a high-level network for clinical research. We have achieved a lot with projects such as the Breast International Group, but mostly because of the enthusiasm of individuals and good collaboration with industry partners. Public funding needs to be increased if we want to maintain and improve our results. 
Q How do you see the future of breast cancer treatment, both surgical \& medical, in the coming 5-10 years?

We will be getting closer to understanding the different properties and optimal treatment approaches of what is no longer one single disease. Nowadays, we can provide cure for three out of four breast cancer patients, but we will have to increase our efforts for the remaining 25\%.

My vision is that no-one should die from breast cancer any longer; this may take longer than 5-10 years, but it is not unrealistic. As always, the field of surgery moves quicker and more substantially than other disciplines: what was a mutilating procedure 20 years ago for most patients will soon be a relatively comfortable outpatient procedure for the majority. What to do, when and for whom, this is going to be the most challenging task we face, particularly in times of limited resources in many societies.

\section{Disclaimer}

The opinions expressed in this interview are those of the interviewee and do not necessarily reflect the views of Future Medicine Ltd.

Financial \& competing interests

\section{disclosure}

$M$ Gnant has no relevant affiliations or financial involvement with any organization or entity with a financial interest in or financial conflict with the subject matter or materials discussed in the manuscript. This includes employment, consultancies, honoraria, stock ownership or options, expert testimony, grants or patents received or pending, or royalties.

No writing assistance was utilized in the production of this manuscript. 